\title{
Detection of Damage during Quasi-Static Loading of a Single Stringer Panel using Passive Thermography and Acoustic Emission
}

\author{
Mr. Joseph N. Zalameda ${ }^{1}$, Dr. William P. Winfree ${ }^{1}$, and Dr. Michael R. Horne ${ }^{2}$ \\ ${ }^{1}$ NASA Langley Research Center, MS231 Hampton, VA 23681 \\ ${ }^{2}$ National Institute of Aerospace, NASA Langley Research Center, MS231 Hampton, VA 23681 \\ E-mail: joseph.n.zalameda@nasa.gov
}

\begin{abstract}
The purpose of this paper is to describe the methodology of measuring damage onset and growth in a composite structure during quasi-static loading using passive thermography and acoustic emission. The early detection and measurement of damage progression is important to understand failure modes. A single stringer panel was subjected to quasi-static loading to induce deformation which resulted in the formation of damage between the stiffener flange and skin. The loading was stopped when damage growth was detected. Passive thermography and acoustic emission were used to detect damage in real-time as a function of the applied load. Of particular interest are the small transient thermographic signals resulting from damage formation which can be challenging to detect, as compared to the persistent passive thermography indications of cyclic fatigue loading. We describe a custom developed thermal inspection system for detection of composite damage during quasi-static loading. The thermal results are compared to a two-dimensional multi-layered thermal simulation based on the quadrupole method. Acoustic emission is used to further characterize the damage by comparing the acoustic emission events with the thermal imagery. Results are compared to ultrasonic measurements to document the damage through-the-thickness.
\end{abstract}

Keywords: composite inspection, thermography, acoustic emission, fiber break, matrix crack, delamination, quasi-static loading

\section{INTRODUCTION}

Real-time nondestructive evaluation (NDE) is necessary for composites load testing to track early onset and growth of damage. The NDE allows for controlling the growth of the damage as a function of the applied load. In this work, a quasi-static bending load (using 7 contact points) is applied to a single stringer stiffened composite panel. When damage is detected with the realtime NDE, the loading is stopped and another inspection technique such as ultrasound or X-ray CT is used to provide a detailed assessment of the panel damage as a function of depth. Documentation of damage onset, growth, and panel failure will provide valuable information for development and validation of progressive damage analysis (PDA) models [1,2]. The ultimate goal is to use the validated PDA models to decrease the time required to test and certify composite materials/designs for aircraft structures and therefore save development costs. The purpose of this paper is to describe the methodology of measuring damage onset and growth in a composite structure during quasi-static loading using passive thermography and acoustic emission.

There have been numerous studies combining thermography with acoustic emission for structures testing with most applications for fatigue loading [3-9]. The challenge for passive thermography is to detect the small transient thermal signals generated by a quasi-static load as compared to the repetitive thermal signals resulting from cyclic loading. Similar to previous studies, we temporally compare the acoustic emission event with the thermal imagery. This allows thermal confirmation that a detected acoustic emission event results in damage onset or growth that would be of interest to characterize using ultrasound or X-ray CT. The thermal anomalies are very small and barely within the detection limits of the infrared camera and therefore a custom data acquisition system was developed to detect the small thermal signatures in real time. Multiple IR cameras and multiple acoustic emission sensors could be employed for large scale testing of structures, however for this effort given the panel size and setup a single thermal camera and six acoustic emission sensors were utilized.

\section{SAMPLE}

The stiffened composite panel skin is 12 plies with a thickness of $0.22 \mathrm{~cm}$, the stiffener flange is 12 plies thick with a thickness of $0.24 \mathrm{~cm}$. The stiffener is a woven composite. The stiffened composite panel flat side, stringer side with acoustic emission locations, the painted specimen, and cross-sectional view are shown in Figs. 1a, 1b, 1c, and 1d respectively. The panel was painted for digital image correlation measurements to record panel deformation. Quasi-static loads were applied using seven application points, two on top (located in middle just outside of the flange) and five on the bottom (located at each corner and 
center). The load was applied from the bottom while the top was held stationary at the two points. This configuration allows for panel deformation that results in damage formation between the stiffener flange and skin. The applied quasi-static loads were up to 1,000 pounds. Passive thermography was used to track damage on the stringer side and the array of acoustic emission sensors were mounted on the skin side. The test setup is shown in Fig. 2a along with infrared camera inspection view shown in Fig. 2b. Examples of the applied load for a typical test and panel setup while under load showing deflection are shown in Figs. $3 a$ and $3 b$ respectively.

(a) Flat Side

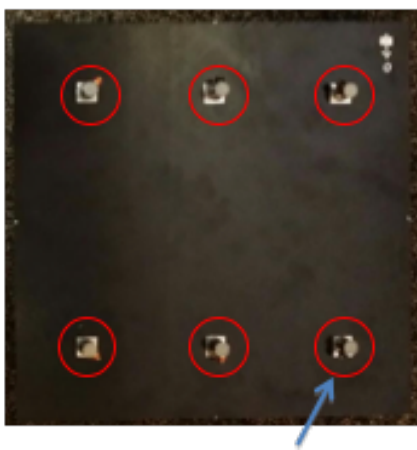

Acoustic Emission Sensors (b) Stringer Side

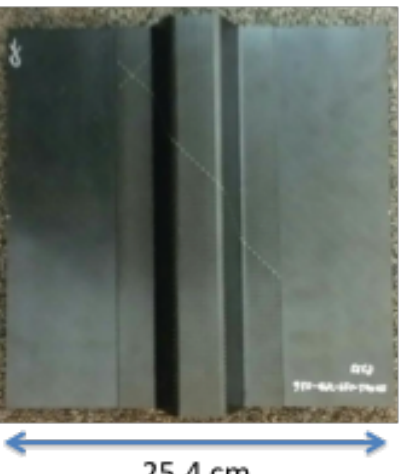

$25.4 \mathrm{~cm}$ (c) Stringer Side (Painted)
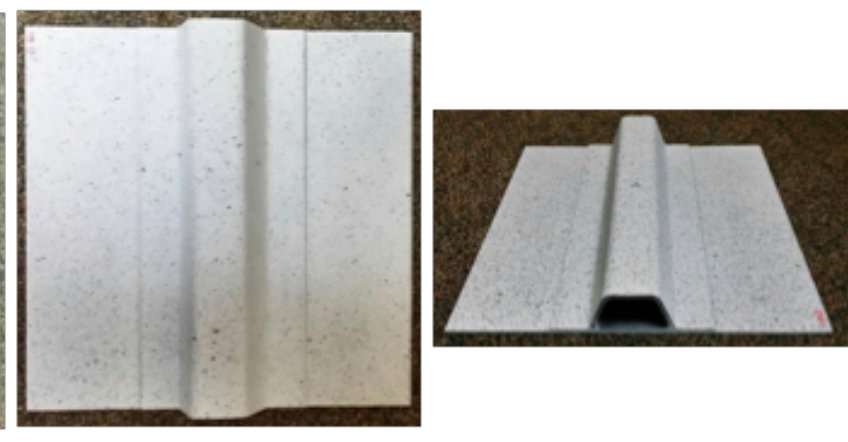

Figure 1: Single stringer composite panel with acoustic emission sensor locations.

(a) Setup

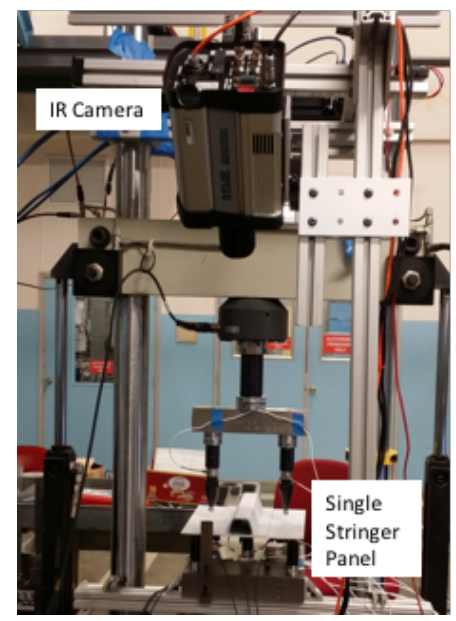

(b) Infrared Camera View

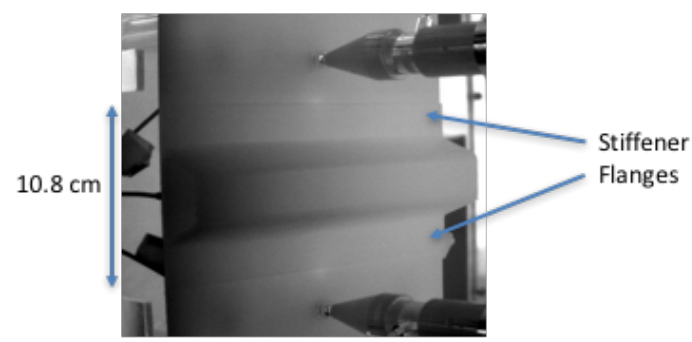

Figure 2: Quasi-static loading setup for single stringer panel with infrared camera view.

\section{INSPECTION SYSTEMS}

The IR camera shown in Fig. 2 operates in the mid-wave 3-5 micrometer band with a dynamic range of 14 bits. The IR camera is configured with a $25 \mathrm{~mm}$ germanium lens. The focal plane array size for the camera is $640 \times 512$. The camera frame rate was 80 to $180 \mathrm{~Hz}$. The IR camera was connected to a custom developed data acquisition system (via GigE interface) for image acquisition. The custom developed data acquisition system, in addition to image acquisition, allowed for simultaneous acquisition of load data, real time processing of the IR imagery using averaging and delayed subtraction, and real time contrast adjustment. The applied load was acquired simultaneously using a USB 12-bit data acquisition module. Both the unprocessed thermal images and applied load were written to a solid state hard drive while displaying the processed imagery. The real-time processing of the displayed thermal images was required to detect the small thermal indications of damage [9,10]. The setup required a Plexiglas ${ }^{\circledR}$ shield (not shown in Fig. 2) to filter out spurious IR background sources. 
(a) Quasi-Static Loading

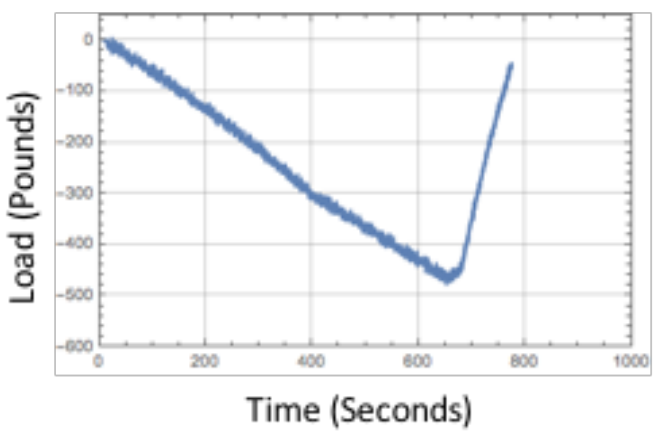

(b) Panel Deformation

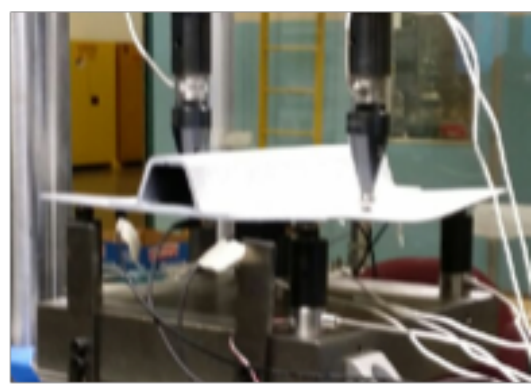

Figure 3: Applied load and panel deformation.

Six acoustic emission sensors were bonded to the composite panel on the skin side (shown in Fig. 1a). Each sensor was connected to a data acquisition computer via Digital Wave TM PA0 preamp/line drivers and a Digital Wave TM FM1 signal conditioning channel amplifier for capture of acoustic data at $2 \mathrm{MHz}$ sample rate and 1024 points per event. The acoustic emission computer also captured the load for synchronization to the thermal images. Acoustic emission systems collect structure-borne sound, typically not audible, in the ultrasonic frequency band (approx. 50 to $500 \mathrm{kHz}$ ), generated by dynamic displacements such as damage initiation and growth at picometer scales [11]. Pre-cursors to failure are monitored in real-time. The total signal energy, $S E$, of an acoustic signal is given as:

$$
S E=\sum_{i=1}^{N} V_{i}^{2} \Delta t
$$

where $V_{i}$ is the signal voltage, $i$ is the time reference point, $N$ is the number of time points in the signal, $\Delta \mathrm{t}$ is the sampling time per point. Trends of the energy over time, or load, are used to identify and track damage development. Before each load test, pencil lead breaks are performed at various locations on the panel to calibrate and test the acoustic emission sensors.

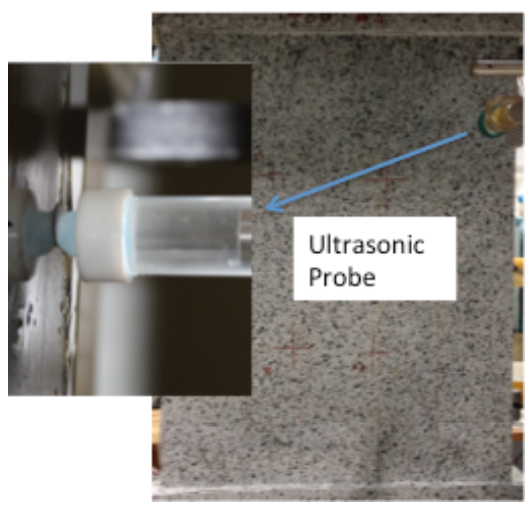

Figure 4: Close up of ultrasonic probe.

Ultrasonic inspections were implemented at intervals determined by the indication of damage growth from thermography and acoustic emission. The ultrasonic inspection technique utilizes a broadband $10 \mathrm{MHz}$ transducer $(1.27 \mathrm{~cm}$ diameter with $5.08 \mathrm{~cm}$ focus) contained in a captive water column with a flexible membrane tip design [12]. Water is sprayed onto the surface for ultrasonic coupling. The probe is scanned over the entire panel on the flat side. A picture of the setup is shown in Fig. 4.

\section{MEASUREMENT RESULTS}

Digital image processing was required to both enhance the detection of thermal events during load and to facilitate comparison of the thermal inspection imagery to the ultrasonic data. To enhance detection in real-time, image averaging and a delayed subtraction algorithm was implemented. Typical parameters of $20-100$ frames were averaged and a delay subtraction of 20 averaged frames were typically used. The delayed subtraction removed fixed background infrared radiation while increasing 
sensitivity to changes. To further remove noise, a de-noising algorithm using singular value decomposition was implemented $[13,14]$. Lastly, an image perspective transformation was used to correct for the infrared camera look angle since the optical line of sight was not normal $[14,15]$. Detected damage progression during load runs 1-4 are shown in Figs. 5, 6, and 7. The infrared image is rotated right so the top stiffener flange is the left flange of Fig. $2 \mathrm{~b}$. The thermal damage was detected over camera noise and background reflections using three criteria: observing a bright area that appears suddenly, stays in one location, and then slowly decays in intensity over time. When damage is detected the loading is stopped, the sample is removed, and an ultrasound inspection is performed to document the damage through-the-thickness. The respective ultrasound inspections are also shown in Figs. 5, 6, and 7. In Fig. 5, for run 1, a small thermal indication (circled in red) was detected and the resulting ultrasonic inspection revealed no damage. This was typical for several of the samples tested. A small thermal indication did not always result in detectable damage using ultrasound. The small indication in Fig. 5 could be a small matrix

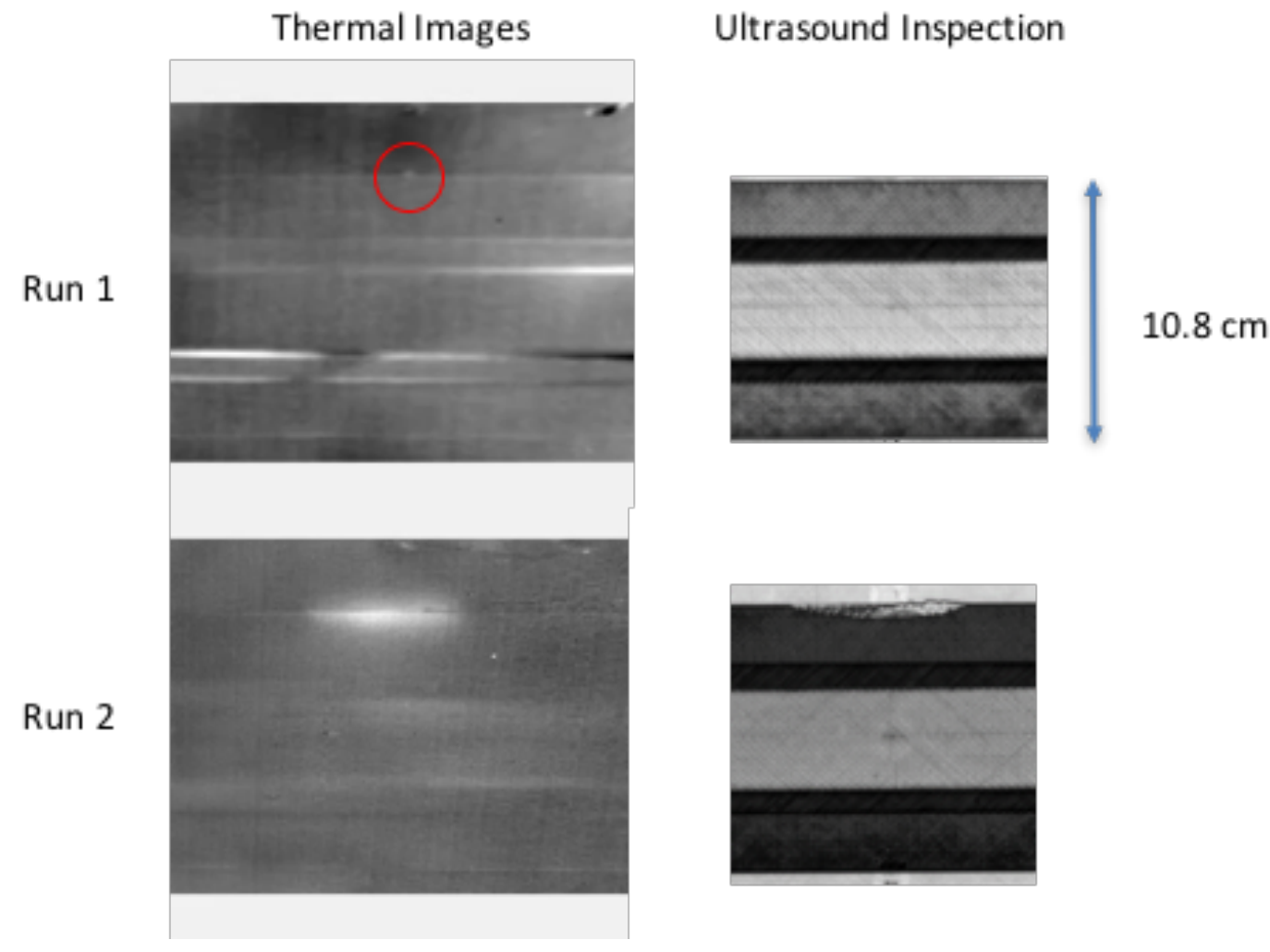

Figure 5: Processed thermal inspection images for quasi-static load runs 1 and 2 with corresponding ultrasound inspection images.

crack or fiber break located at the stiffener flange edge perhaps beyond the detection limits of ultrasound. Also, any damage at this edge interface would be difficult to detect with ultrasound especially at the corner. X-ray CT inspection could be used to help understand these initial small thermal indications. The run 2 thermal image in Fig. 5 clearly shows a delamination at the stiffener flange edge. The thermal energy release due to the disbond is sufficient to generate a temperature increase sufficient to provide the general shape of the damage. The ultrasound inspection image is in agreement with the thermal indication. For load run 3 it is interesting to note the delamination growth, as indicated by the ultrasound inspection image, did not produce a sufficient temperature increase to resolve the shape and size of the damage. This is due, in all likelihood, to the delamination growing in increments from one side to another. This is plausible since the small thermal indications (circled) were detected at opposite sides of the delamination at different times as shown in Fig. 6. Shown in Fig. 7 is the final load run 4 where a large delamination was detected at the bottom as shown in the ultrasound inspection image. From the thermal inspection imagery, the top delamination appeared to grow only slightly on the left side. From the ultrasonic image, the top delamination appeared to grow slightly and then result in a sudden bottom failure. At 3.33 seconds, there appears to be a single layer failure (circled) at the skin leading into the stiffener flange. At 3.88 seconds, there is a large thermal indication that appears showing the overall shape of the delamination with good agreement as shown by the ultrasound inspection. 
Run 3
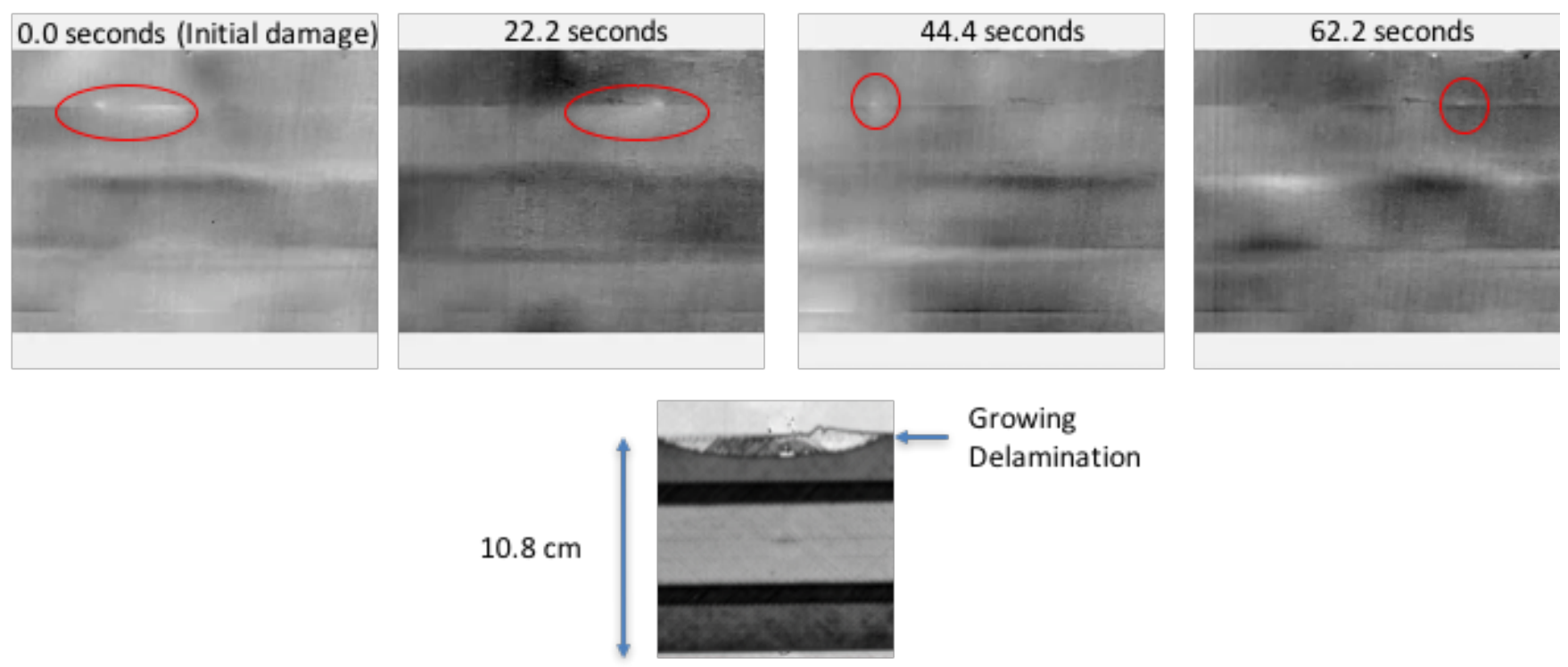

Ultrasound Inspection

Figure 6: Processed thermal inspection images for quasi-static load run 3 with corresponding ultrasound inspection image.

Run 4
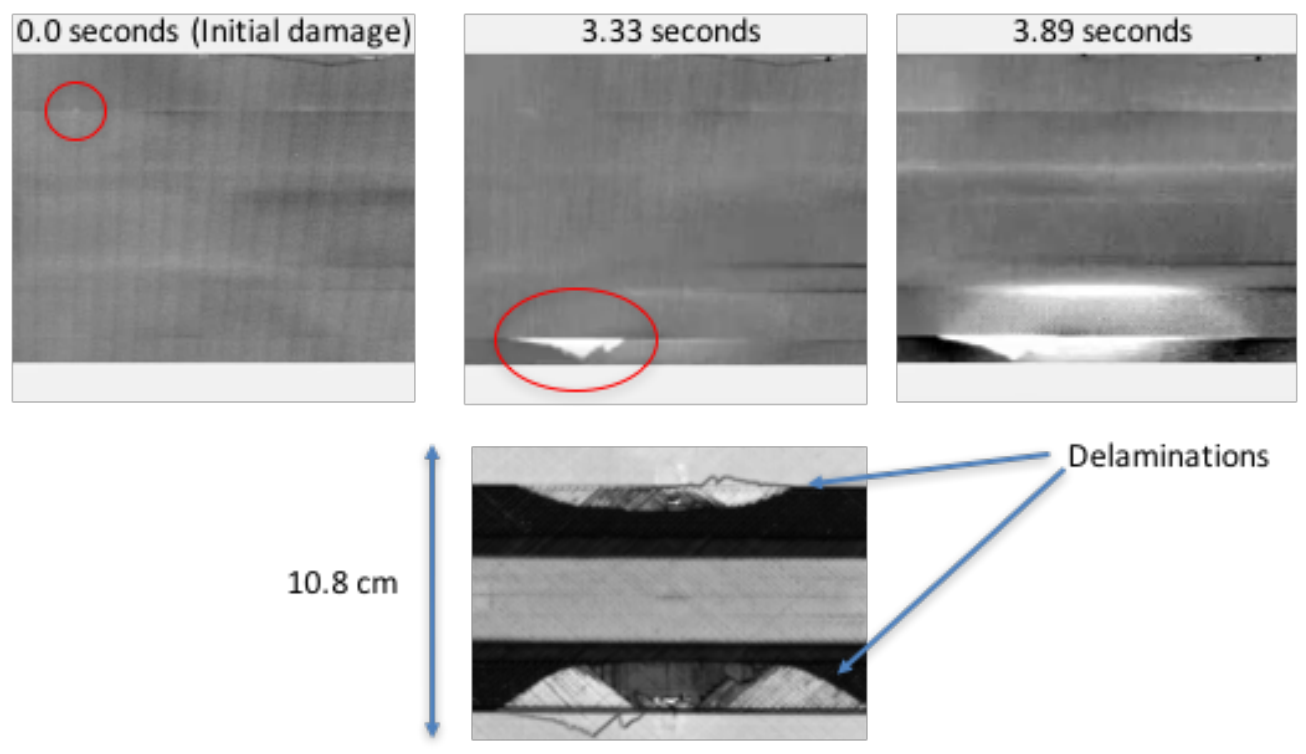

Ultrasound Inspection

Figure 7: Processed thermal inspection images for quasi-static load run 4 with corresponding ultrasound inspection image.

\section{THERMAL MODELING}

A two-dimensional thermal model is studied using the quadrupole method presented previously in a paper by Winfree and verified by comparison to finite element modeling [16]. The advantages of the quadrupole method are: easily extendable to multiple layers, faster computation time as compared to finite element method simulations, and easy to insert realistically shaped flaws into simulations [17]. For this case, with no convection losses at the vertical or horizontal edges, the heat flux 
generated is at the flaw location within the structure, as shown in Fig. 8. For static loading scenarios, the heat flux is produced during damage formation and has been observed to be instantaneous. A two-layer configuration with a finite lateral dimension is shown in Fig. 8 with no heat flow across the vertical edges at $\mathrm{x}=0$ and $\mathrm{x}=\mathrm{L}$.

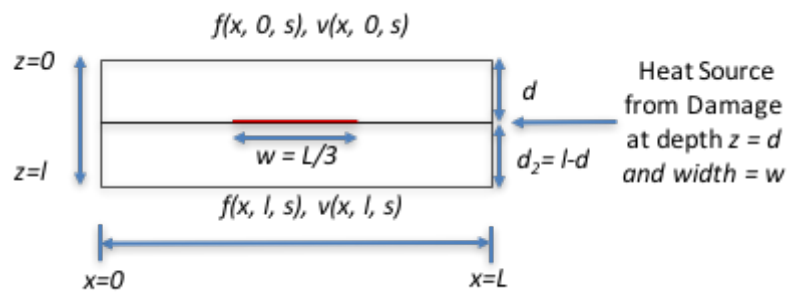

Figure 8: Configuration for two-dimensional model with embedded heat source.

The condition of no heat flow across the vertical edges is satisfied if the solution in the $\mathrm{x}$-direction is given by a cosine transform. Each of the coefficients of the cosine transform of the two-dimensional Laplace transform temperature solution can be found by dividing the layer in two layers of thickness $d$ and $l-d$ each with a matrix form given as:

$$
\begin{gathered}
{\left[\begin{array}{c}
\tilde{v}_{m}(d, s) \\
\tilde{f}_{m}(d, s)-\tilde{f}_{m}^{s} / 2
\end{array}\right]=\left[\begin{array}{cc}
\cosh \left[q_{m} z\right] & -\frac{1}{K q_{m}} \sinh \left[q_{m} z\right] \\
-K q_{m} \sinh \left[q_{m} z\right] & \cosh \left[q_{m} z\right]
\end{array}\right]\left[\begin{array}{c}
\tilde{v}_{m}(0, s) \\
\tilde{f}_{m}(0, s)
\end{array}\right],} \\
{\left[\begin{array}{c}
\tilde{v}_{m}(l, s) \\
\tilde{f}_{m}(l, s)
\end{array}\right]=\left[\begin{array}{cc}
\cosh \left[q_{m}(l-d)\right] & -\frac{1}{K q_{m}} \sinh \left[q_{m}(l-d)\right] \\
-K q_{m} \sinh \left[q_{m}(l-d)\right] & \cosh \left[q_{m}(l-d)\right]
\end{array}\right]\left[\begin{array}{c}
\tilde{v}_{m}(d, s) \\
\tilde{f}_{m}(d, s)+\tilde{f}_{m}^{s} / 2
\end{array}\right],} \\
\text { where } q_{m}=\sqrt{\frac{s}{\alpha_{z}}+\frac{\alpha_{x}}{\alpha_{z}}\left(\frac{\pi m}{L}\right)^{2}},
\end{gathered}
$$

and $\tilde{v}_{m}(z, s)$ and $\tilde{f}_{m}(z, s)$ are the cosine coefficients for the temperature and flux respectively and $\tilde{f}_{m}^{s}$ is the cosine coefficient for the source flux. The Laplace transform of the surface temperature and flux are given as $\tilde{v}_{m}(0, s)$ and $\tilde{f}_{m}(0, s)$ respectively. In addition, $K$ is the composite thermal conductivity, $\alpha_{z}$ is the thermal diffusivity in the z direction, $\alpha_{x}$ is the thermal diffusivity in the $\mathrm{x}$ direction, $s$ is the Laplace complex argument, $l$ is the slab thickness, $d$ is the depth of the flaw, $m$ is the Fourier cosine series coefficients for each lateral index of $\mathrm{x}$, and $L$ is the slab width. Given damage at depth $z=d$ and no heat flow at the surface, equations (2) and (3) can be solved to find the temperature at the surface to be as:

$$
\tilde{v}_{m}(0, s)=\frac{f_{m}^{S} \cosh \left[q_{m}(l-d)\right]}{K q_{m} \sinh \left[q_{m} l\right]}
$$

Equation (5) can be solved by taking into account the discrete positions in $\mathrm{x}$ by the vector representation:

$$
\boldsymbol{V}(0, s)=\boldsymbol{C}(s) \cdot \boldsymbol{F}^{s}(s)
$$

where the elements of $\boldsymbol{C}(s)$ are given as:

$$
C_{i, j}(s)=a_{j} \frac{\sum_{m=0}^{N-1} a_{m} \cos \left(\frac{i m \pi}{N-1}\right) \cos \left(\frac{j m \pi}{N-1}\right) \cosh \left[q_{m}(l-d)\right]}{(2 N-2) K q_{m} \sinh \left[q_{m} l\right]} .
$$


Both $a_{j}$ and $a_{m}$ are equal to 1 if the index $\mathrm{j}$ (or $\left.\mathrm{m}\right)=0$ or N-1 and 2 otherwise. The heat flux vector, $\boldsymbol{F}^{\boldsymbol{s}}(s)$, is a vector representation of the heat generated during the formation of the flaw during loading at depth $\mathrm{z}=\mathrm{d}$ and is modeled as an impulse function. For each point, the inverse Laplace transform is calculated at a given time to give temperature as a function of $x$ position. The inverse Laplace transform for the surface temperature, $\boldsymbol{V}(0, s)$, is calculated using the Talbot inversion algorithm [18]. Typical results are shown in Figs. 9a, 9b, and 10 for maximum temperature as a function of flaw depth, blurring as a function of flaw depth for the maximum temperature and blurring as a function of time for a flaw depth of $0.24 \mathrm{~cm}$ respectively. For these plots, the values used were $\alpha_{z}=0.00425 \mathrm{~cm}^{2} / \mathrm{sec}, \alpha_{x}=0.0425 \mathrm{~cm}^{2} / \mathrm{sec}, l$ (the slab thickness) $=0.46 \mathrm{~cm}, K=0.00883$ $\mathrm{W} / \mathrm{cm} / \mathrm{K}$, the $F l u x=1 \mathrm{Watt} / \mathrm{cm}^{2}$, flaw width set from 2.5 to $5.0 \mathrm{~cm}$, and $L$ the slab width set to $7.6 \mathrm{~cm}$. As can be seen from Fig. 9a, there is considerable temperature drop for sources that are deeper. This is especially problematic if one takes into account camera noise. Additionally, for deep sources, there is considerable blurring of the edges of the source as shown in Fig. 9b. There is also considerable blurring over time as shown in Fig. 10. This indicates that the earliest detectable thermal signal will give the best sizing of the defect.

(a)

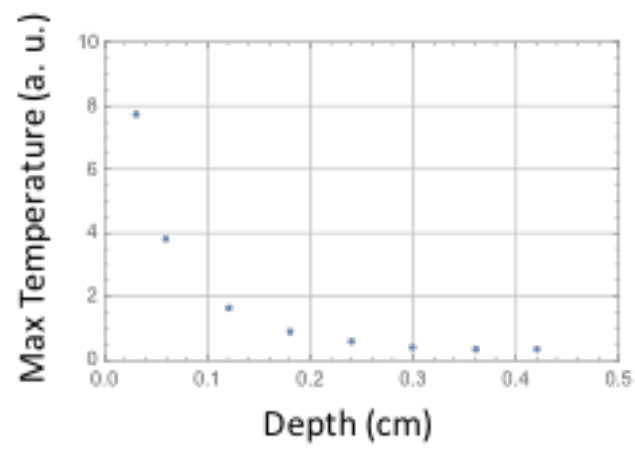

(b)

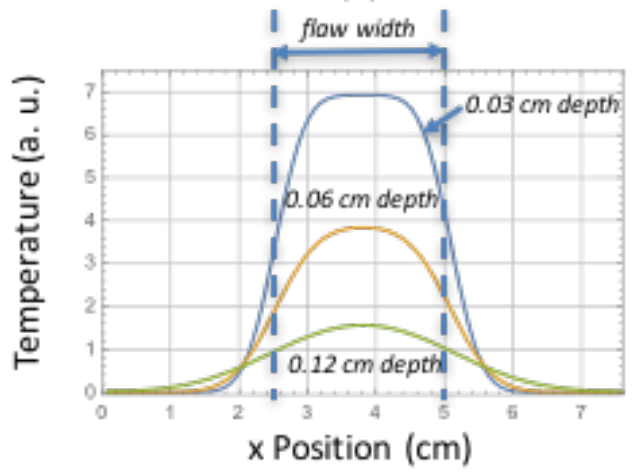

Figure 9: Temperature drop as a function of source depth and blurring of source edge for different depths.

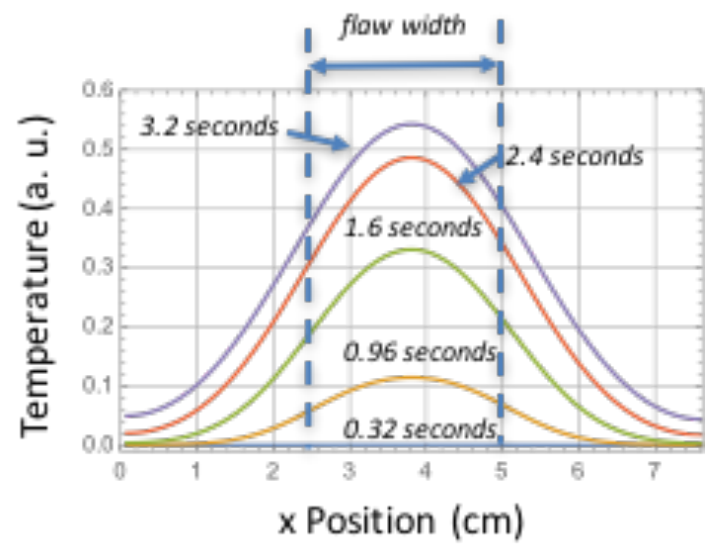

Figure 10: Temperature as a function of time for a flaw source buried $0.24 \mathrm{~cm}$.

\section{THERMAL IMAGERY COMPARISON TO ACOUSTIC EMISSION}

Acoustic emission data were collected during each quasi-static load test. When a sufficient number of acoustic emission events clustered in a short period of time were detected, this represents rapid damage formation and the loading was stopped to characterize the damage. A typical load profile along with the detected acoustic emission events are shown in Fig. 11. The acoustic emission is sensitive to fiber breaks and matrix cracks and these events can produce heat. Shown in Fig. 12, are the corresponding processed thermal images. The thermal images show heating resulting from a small edge delamination growing along the edge of the stiffener flange. This is shown in the ultrasound results in Fig. 12. This is a successful capture of an early delamination formation that can be grown and characterized during subsequent load runs. The acoustic emission data was compared to thermal data by aligning the recorded load profiles from each system. The results are shown in Table 1, for 


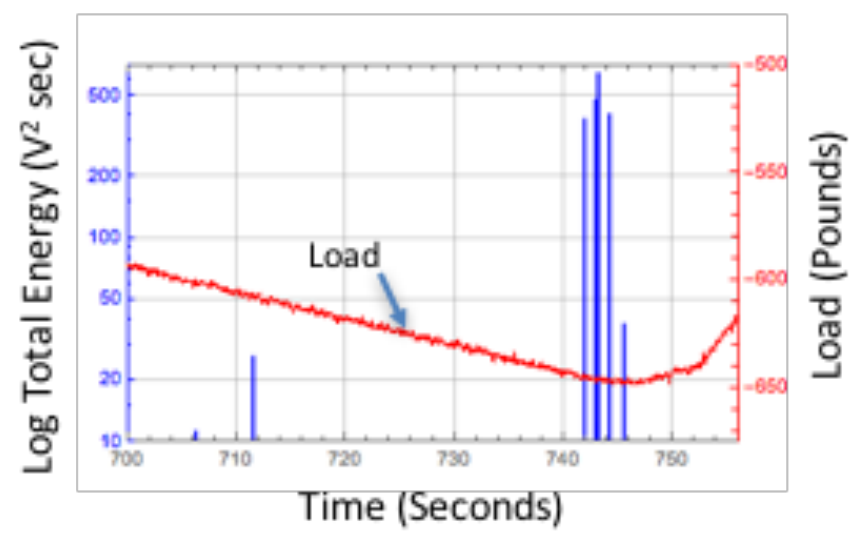

Figure 11: Quasi-static load profile with detected acoustic emission events.

a given acoustic energy event (greater than 10 volts $^{2}$ second), the acoustic emission event time is compared to the thermal image indication time. The thermal image indication time is determined from the earliest thermal indication where new heating is observed. This can be seen in Fig. 12 at 707.0 seconds a small thermal indication is observed and grows as a function of time as the applied load increases. Table 1 provides a comparison of the acoustic emission time to the thermal indication time. As shown in Table 1 most acoustic emission events corresponded to a detected thermal indication. This shows the sensitivity

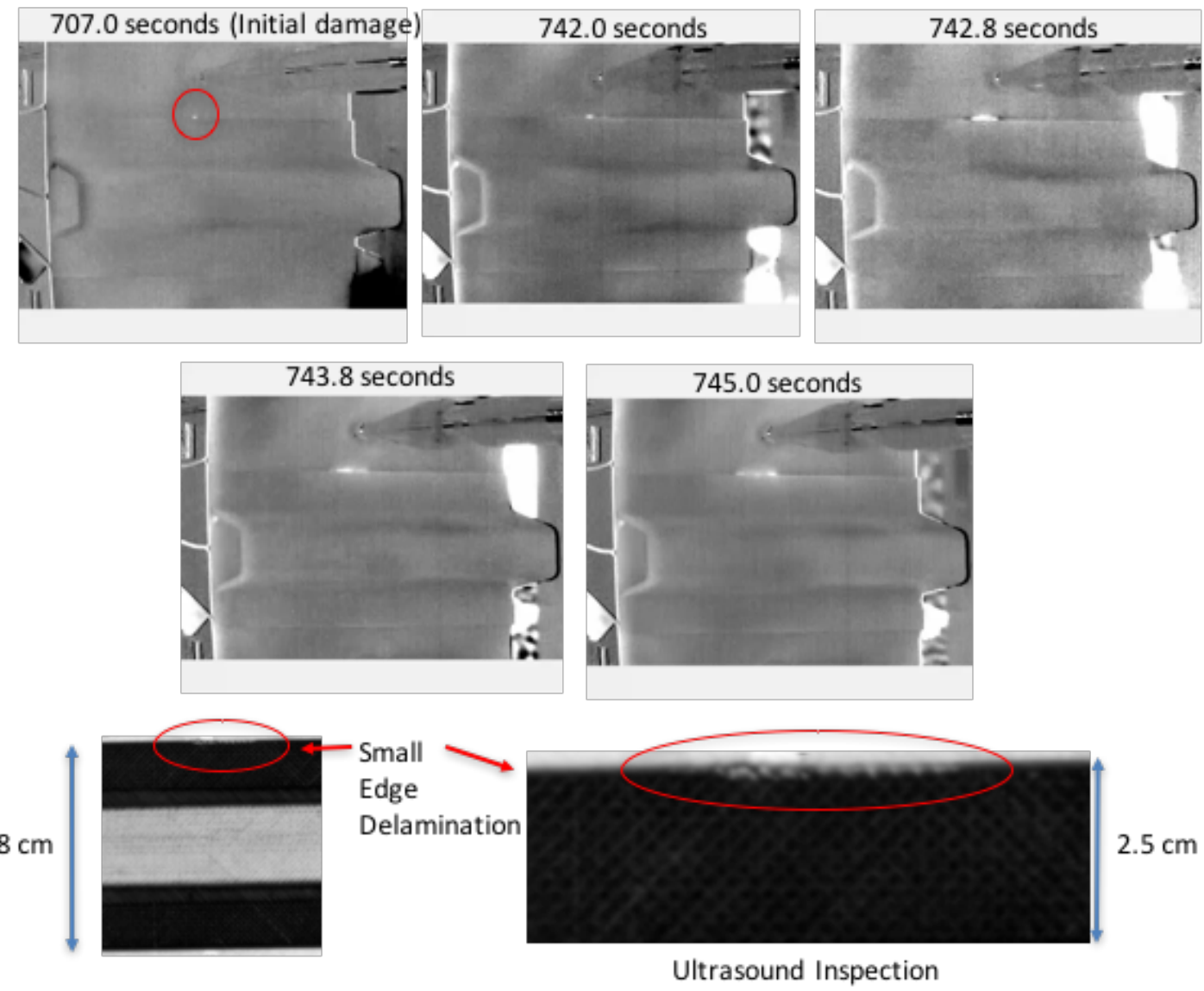

Figure 12: Processed thermal inspection images for a single quasi-static load run with corresponding ultrasound inspection image showing edge delamination. 
of the thermal technique to detect and locate the damage. This was helpful since acoustic emission, for these tests and panel setup was not able to determine damage location. For the acoustic emission event at time 711.5 seconds there was no thermal indication detected. This could be due to the damage located underneath the hat which would be out of the field of view of the infrared camera or a fiber break/matrix crack located at a significant depth that would be undetectable. Nevertheless, thermal imaging provides good agreement with acoustic emission.

Table 1: Comparison of acoustic emission event time to thermal image indication time.

\begin{tabular}{|c|c|c|}
\hline $\begin{array}{c}\text { Acoustic } \\
\text { Emission Event Energy (Volts } \\
\text { Second) }\end{array}$ & $\begin{array}{c}\text { Acoustic } \\
\text { Emission Event Time } \\
\text { (Seconds) }\end{array}$ & $\begin{array}{c}\text { Thermal Image Indication } \\
\text { Time (Seconds) }\end{array}$ \\
\hline 11.4 & 706.2 & 707.0 \\
\hline 26.5 & 711.5 & Nothing Detected \\
\hline 389.7 & 741.9 & 742.0 \\
\hline 483.7 & 742.9 & 743.3 \\
\hline 653.6 & 743.1 & 744.6 \\
\hline 411.0 & 744.2 & 745.9 \\
\hline 38.3 & 745.5 & 747.2 \\
\hline
\end{tabular}

The data in Table 1 also demonstrates a consistent time delay between the acoustic emission event time and the thermal indication time. This is expected since acoustic emission detection time would be nearly instantaneous as compared to the thermal detection time. The thermal detection time requires the diffusion of heat to the surface. Another way to compare acoustic emission to passive thermography is count the number of pixels above a threshold. This is shown in Fig. 13 where the most significant acoustic emission events (energy $>100$ volts $^{2}$ second) are compared to the thermally detected area pixels (pixel values greater than 7 counts shown in dashed line plot) as a function of time. What this clearly shows is the acoustic emission event occurs before a growth in thermally detected damage area. A failure generates an acoustic emission event and heat will take time to diffuse through-the-thickness as well as in-plane.

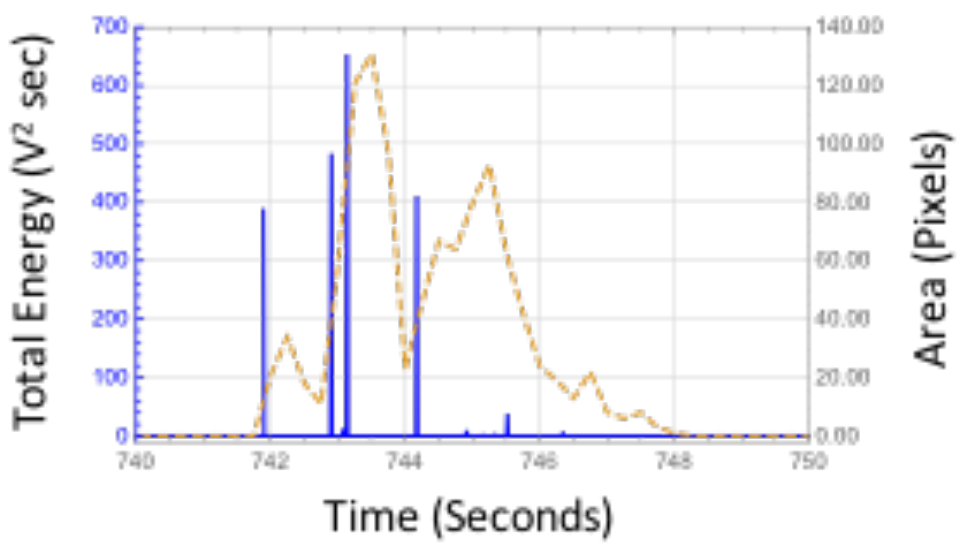

Figure 13: Detected acoustic emission events compared to thermally detected area pixels above a threshold.

\section{CONCLUSIONS}

It has been shown that passive thermography can be used to detect damage in hat stiffened composite structures during quasistatic loading. The thermally detected damage shape is in good qualitative agreement with the ultrasonic inspection results. In addition, it appears, small thermally detected damage can be detected that are beyond the limits of detection with conventional 
ultrasound. This is likely a result of the significant loss of ultrasonic signal at the edge of stiffener due to the ultrasonic beam straddling a significant thickness change. The thermal results compared well to the acoustic emission with most acoustic emission events corresponded to a thermal indication thus allowing acoustic emission event location. The obvious limitation is the damage must be in the field of view of the thermal imager and at a depth that would allow detection within the detectability limits of the infrared camera. A second thermal camera located on the opposite side (skin side) would be valuable to capture damage that could be hidden by the stiffener hat and could capture the deeper damage facing the opposite side camera. Lastly, a more careful study of the acoustic emission event time compared to the temporal response of the detected thermal transient might provide a start time that would allow fitting of a thermal model to determine damage depth in real-time. This would provide damage location, size and depth.

\section{REFERENCES}

[1] O'Brien, T. K., "Development of a Delamination Fatigue Methodology for Composite Rotorcraft Structure", NASA Aviation Safety Technical Conference Denver, CO, (October 2008).

[2] Krueger, R., "An Approach to Assess Delamination Propagation Simulation Capabilities in Commercial Finite Element Codes", NASA/TM-2008-215123, (2008).

[3] Bisagni, C., Dávila, C. G., Rose, C., and Zalameda, J. N., "Experimental Evaluation of Damage Progression in Postbuckled Single Stiffener Composite Specimens", American Society for Composites $29^{\text {th }}$ Technical Conference Proceedings, USJapan 16, ASTM D30, (Feb. 2014).

[4] Zalameda, J. N., Burke, E. R., Parker, R. F., Seebo, J. P., Wright, C. W., and Bly, J. B., "Thermography Inspection for Early Detection of Composite Damage in Structures during Fatigue Loading", Thermosense XXXIV, edited by Douglas Burleigh, Gregory R. Stockton, Proc. of SPIE Vol. 835403 (April 2012).

[5] La Rosa, G., Clienti, T., Savio, F. Lo, "Fatigue Analysis by Acoustic Emission and Thermographic Techniques", Procedia Engineering, XVII International Colloquium on Mechanical Fatigue of Metals, (ICMFM17), Volume 74, pp. 261-268, (2014).

[6] E. Kordatos, K. Dassios, D. Aggelis, T. Matikas, "Rapid evaluation of the fatigue limit in composites using infrared lockin thermography and acoustic emission", Mechanics Research Communications, 54, pp. 14-20, (2013).

[7] Munoz, V., Vales, B., Perrin, M., Pastor, M. L., Welemane, H., Cantarel, A., and Karama, M., "Damage detection in CFRP by coupling acoustic emission and infrared thermography", Composites Part B: Engineering, Volume 85, pp. 68-75, (February 2015).

[8] Ringermacher H. I., et al, "System and Method for Locating Failure Events in Samples Under Load", United States Patent No. 7,516,663 B2, (April 14, 2009).

[9] Zalameda, J. N., Burke, E. R., Horne, M. R., and Madaras, E. I., "Large Area Nondestructive Evaluation of a Fatigue Loaded Composite Structure", Residual Stress, Thermomechanics \& Infrared Imaging, Hybrid Techniques and Inverse Problems, Vol. 9, Proceedings of the 2016 Annual Conference on Experimental and Applied Mechanics, ISBN 978-31942254-1, Chapter 4, (2017).

[10] Winfree, W. P., Zalameda, J. N., and Howell, P. A., "Improved flaw detection and characterization with difference thermography", Proc. SPIE 8013, 80130U (April 2011).

[11]Horne, M. R. "Rayleigh Wave Acoustic Emission during Crack Propagation in Steel". PhD Dissertation, Virginia Polytechnic Institute and State University. (2003).

[12] Johnston, P. H., Wright, C. W., Zalameda, J. N., and Seebo, J. P., "Ultrasonic monitoring of ply crack and delamination formation in composite tube under torsion load", Ultrasonic Symposium (IUS), IEEE, pp. 595 - 598, (2010).

[13] Hourigan, J. S., and McIndoo, L. V., "The Singular Value Decomposition.” Dec. 1998. College of the Redwoods.

[14]Zalameda, J. N. and Horne, M. R., "Real Time Detection of Damage during Quasi-Static Loading of a Single Stringer Panel using Passive Thermography", Thermosense XL, edited by Douglas Burleigh, Jaap deVries, to be published Proc. of SPIE (April 2018).

[15]Chan, M., (published 2012, March 09), "Perspective Control/Correction", Retrieved from URL https://www.mathworks.com/matlabcentral/fileexchange/35531-perspective-control--correction (downloaded Jan. 2018).

[16] Winfree, W. P., Zalameda, J. N., and Gregory E. D., "Application of the Quadrupole Method for Simulation of Passive Thermography", Proc. SPIE 10214, Thermosense: Thermal Infrared Applications XXXIX, 102140Y (5 May 2017).

[17] Winfree, W. P., Zalameda, J. N., Howell, P. A., and Cramer K. E., "Simulation of Thermographic Responses of Delaminations in Composites with Quadrupole Method", Proc. SPIE 9861, Thermosense: Thermal Infrared Applications XXXVIII, 98610N (2016).

[18] Talbot, A., IMA J Appl Math 23 (1): 97-120, (1979). 